\title{
Degradation Kinetics of Acid-Sensitive Hydrogels
}

\author{
Soeun Kim, Olga Linker, Kim Garth, and Kenneth R. Carter* \\ Department of Polymer Science and Engineering, Conte Center for Polymer Research, University of \\ Massachusetts- Amherst, Governors Drive, Amherst, Massachusetts 01003, United States \\ *The correspondence should be addressed. E-mail: krcarter@polysci.umass.edu
}

\begin{abstract}
:
Dimethacrylate or divinyl-functionalized acetal-based crosslinkers were synthesized as building elements of acid-sensitive crosslinked hydrogels. Each crosslinker was prepared under catalytic acidic conditions with different functional groups installed at the acetal position. The hydrophilicity of the crosslinkers was tuned to control acidic-hydrolysis rate. We report the synthesis of hydroxyethyl dimethacrylatefunctionalized dimethyl ketal (CL1), meta- or para-methoxybenzaldehyde based acetals (CL2m and CL2p), poly(ethylene glycol) dimethacrylate-functionalized dimethyl ketal-based crosslinker (CL3), and divinyl-functionalized meta-methoxybenzaldehyde-based acetal crosslinker (V-CL2m). An examination of acetal hydrolysis kinetics of the monomers was performed in aqueous buffer solutions using ${ }^{1} \mathrm{H}$ NMR (proton nuclear magnetic resonance) and UV-Vis (ultraviolet-visible) spectroscopy at various $\mathrm{pH}$ ranges. The hydrolysis rates were strongly dependent on the structure of the acetal. Network films containing CL2m were prepared by thermally initiated polymerization with either hydroxyethylmethacrylate (HEMA) or methylmethacrylate (MMA). A study of the hydrolysis kinetics of these crosslinked films was performed using GC-MS (gas chromatography and mass spectroscopy) to understand the effect of monomer hydrophilicity, crosslinking density, and polymerization mechanism at different pHs. The crosslinked films composed of the hydrophilic monomer, HEMA, show faster hydrolysis than those containing more hydrophobic monomers (e.g. MMA). The hydrolysis rate decreases as the crosslinking density increases. In the case of thiol-ene networks formed by reacting pentaerythritol tetrakis(3mercaptopropionate) and V-CL2m, each repeating unit is composed of an acid-degradable acetal-moiety. Hydrolysis of the thio-ene network films results in depolymerization into two lower molecular weight components, pentaerythritol tetrakis(3-(6-hydroxyhexylthio)propanoate) and meta-methoxybenzaldehyde.
\end{abstract}

Keywords: acetal; hydrolysis; degradation kinetics; degradable crosslinker; thiol-ene networks; GC-MS; acid sensitive 


\section{Introduction}

pH-sensitive polymers or gels have been intensively studied among the stimuli-responsive systems that can show spontaneous physical or chemical changes in response to small external stimuli such as temperature, mechanical stress, and ionic strength. These $\mathrm{pH}$-sensitive polymer systems can be used in bio-related areas such as in drug-delivery systems (DDS), as well as diagnostic and sensing applications because of the various $\mathrm{pH}$ ranges found in human organs and $\mathrm{pH}$ differences between normal tissue and tumor tissue. ${ }^{1-6}$ For example, using changes in $\mathrm{pH}$ as the external stimuli can drive reversible changes in volume by tuning the ionization states of polyelectrolytes or to irreversibly change solubility via cleavage of acid-degradable crosslinked positions in the crosslinked or linear polymer. ${ }^{7}$ Polymers containing acid-degradable linkages have also been exploited in lithographic patterning where high temperature or strong acids are often required to strip the resist after patterning. ${ }^{8-10}$ The use of these acid-sensitive materials not provide protection of the silicon substrate from undesired damage but also leads to milder condition for post-process substrate cleaning and hence lower production costs.

There are a variety of acid-degradable systems based on chemistries including tertiary esters, orthoesters, acetal/ketals, imines, hydrazones, and cis-aconityls - many of these are also frequently used as protective groups in organic synthesis. ${ }^{7}$ For example, polystyrene based nanogels crosslinked by tertiary ester dimethacrylates have been reported and their degradation was demonstrated by treating the samples at $90{ }^{\circ} \mathrm{C}$ for $24 \mathrm{hr}$ leading to their conversion from spherical gel particles to soluble linear polymer. ${ }^{11}$ Acid-sensitive brush polymers were grown from silicon substrates utilizing a tertiary ester-based tethering group. Layers with different brush thickness were demonstrated and these brushes could be easily removed by treatment with aqueous media. ${ }^{12}$ Among the many acid-sensitive functional groups, acetal based crosslinkers 
have various advantages including ease of synthesis, useful hydrolysis at room temperature that occurs within reasonable time at various acidic $\mathrm{pH}$ ranges, stability at neutral or mildly alkaline conditions, and versatile tuning of degradation rate by changing the substituent group at the acetal position.

The degradation rates of hydrogels or polymers which are covalently bonded with aciddegradable monomers have been studied using a variety of methods. Hydrophilic water-soluble materials can be easily characterized in water-based $\mathrm{pH}$ buffer solutions without any complications due to diffusion. These measurements can be made using ${ }^{1} \mathrm{H}$ NMR by observing the disappearance of proton signal corresponding to acid-sensitive functional groups, ${ }^{13}$ or by tracking molecular weight changes using gel permeation chromatography (GPC). ${ }^{14}$ Aciddegradable linear polymers have been synthesized via step-polymerization involving the Michael addition reaction with acetal containing monomers, ${ }^{13}$ direct acetal forming polymerization using diols with divinyl ether ${ }^{15}$ or acyclic diene metathesis (ADMAT) from divinyl monomers with Grubbs' catalyst. ${ }^{2}$ All of these procedures require long reaction times, high monomer purity, and precise control of stoichiometry for high molecular weight. Most linear step-growth polymers will not retain their shape after degradation because the resulting products consist of smaller molecules which are be easily washed away after hydrolysis. In the case of materials crosslinked by simple radical polymerization, the monomers themselves can be selected from those having more than one polymerizable group. After hydrolysis only the crosslinker units will be severed while the linear portion of the polymer remains mainly intact. This can lead to partial shape retention and lends utility of these types of materials to be used in molding operations where the networks can be formed by thermal or UV-initiated polymerization. For these reasons and others, acid-degradable crosslinked films are ideal candidates for the development of self-exfoliating 
garments, which can facilitate the selective removal of contaminated areas by acidic stimuli but maintain its overall original integrity. The $\mathrm{pH}$ range which triggers this degradation could be specifically varied depending on external stimuli.

While networks that controllably degrade in the presence of external stimuli have many potential applications, there has been little activity in studying the acid-catalyzed hydrolysis kinetics of ketal and acetal functional groups present in network films. ${ }^{16}$ It is challenging to quantitatively measure how quickly acid-sensitive groups can be hydrolyzed in crosslinked systems, mainly due to the slow diffusion of aqueous buffer solutions and the relatively small mass loss during the decomposition of the crosslinker units. In the case of acid-sensitive nano- or micro-gel particles, the degradation rate can be indirectly examined by observing particle size changes, for example by using dynamic light scattering (DLS) while taking into account any swelling effect. ${ }^{17}$ The hydrolysis of acid-degradable crosslinked polymer films can be discontinuously measured by monitoring fractional mass loss by repeatedly incubating gels in buffer solution and drying. ${ }^{16}$ However, if the mass loss during hydrolysis is very small, it can be very difficult to measure the mass change after each degradation cycle.

Herein, we report the synthesis and characterization of novel, acid sensitive methacrylatefunctionalized acetal and ketal-based crosslinker monomers. We use ${ }^{1} \mathrm{H}$ NMR and UV-Vis spectroscopy to perform a measurement of the kinetics of their room temperature hydrolysis. Acid-degradable network films composed of hydrophilic HEMA or hydrophobic MMA with the synthesized crosslinkers were prepared and their degradation kinetics were investigated using GC-MS. This paper provides facile way to prepare acid-sensitive crosslinkers and by performing an in-depth measurement of hydrolysis kinetics we provide a new understanding how to control the rate of network decomposition. 


\section{Experimental}

\subsection{Materials and Instruments.}

Hydroxyethylmethacrylate (HEMA, Acros Organics, 97\%), methyl methacrylate (MMA, Acros Organics, 99\%), poly(ethylene glycol) methacrylate (PEG-MA, average $\mathrm{M}_{\mathrm{n}}$ : $360 \mathrm{~g} / \mathrm{mol}$, Aldrich), 2,2-dimethoxypropane (DMP, Acros Organics, 98\%), p-toluenesulfonic acid

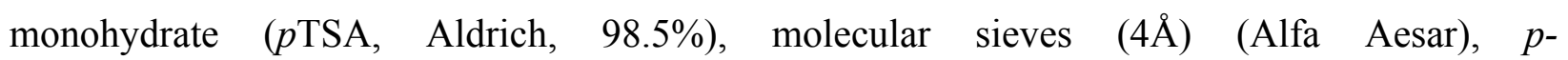
methoxybenzaldehyde ( $p$ MBA, Alfa Aesar, 98\%), m-methoxybenzaldehyde ( $m$ MBA, Alfa Aesar, 98\%), 5-hexen-1-ol (TCI, 95\%), triethylamine (TEA, Acros Organics, 99\%), anhydrous tetrahydrofuran (THF) (EMD chemicals, 99.9\%), cyclohexane (Fisher Scientific, 99\%), pentaerythritol tetrakis(3-mercaptopropionate) (PTMPA, Aldrich, 95\%), pH buffer solution (pH1 5, Fluka) were used without further purification. Azobisisobutyrontitrile (AIBN, Aldrich, 98\%) was recrystallized before use.

${ }^{1} \mathrm{H}$ and ${ }^{13} \mathrm{C}$ NMR spectra were recorded on a Bruker Avance $400(400 \mathrm{MHz})$ spectrometer. Chemical shifts were given relative to residual solvent peaks in deuterated solvents, chloroform $\left(\mathrm{CDCl}_{3}\right)$ and deuterated oxide $\left(\mathrm{D}_{2} \mathrm{O}\right)$. All the NMR data for confirmation of synthesis are available in supporting information (S2 S8). UV-Vis spectroscopy were recorded on a Cary 50 $\mathrm{UV}-\mathrm{Vis}$ absorption spectrometer with $1 \mathrm{~cm}$ path length quartz cuvettes at room temperature. GCMS was measured using a HP 5890 GC-MS (Agilent DB-5ms column consisting of a fused silica capillary, $30 \mathrm{~m}$ length, $0.2 \mathrm{~mm}$ inner diameter and $0.325 \mu \mathrm{m}$ film thickness) injecting $2 \mu \mathrm{L}$ of dilute solution and ramping from room temperature to $350{ }^{\circ} \mathrm{C}$. 


\subsection{Synthesis of crosslinkers}

CL1: Synthesis based on a literature procedure was followed and optimized. ${ }^{9}$ In a $250 \mathrm{~mL}$ round bottom flask, $0.55 \mathrm{~g}$ of $p$ TSA (2.91 mmol, 0.07 equiv.) were dissolved in THF and molecular sieves were then added to the solution. After $15 \mathrm{~min}, 13.53 \mathrm{~g}$ HEMA (104 mmol, 2.5 equiv.) and $4.3 \mathrm{~g}$ 2,2-DMP (41.6 mmol, 1 equiv.) were added and the mixture was stirred for 6 hours at room temperature. The reaction was quenched by adding $\sim 3 \mathrm{~mL}$ TEA $(\mathrm{pH}>7)$, the molecular sieves were removed by filtration and solvent was evaporated in vacuum. After removing solvent, the transparent liquid residue was purified by column chromatography with silica gel (ethyl acetate:hexane:TEA=1:9:0.1, v/v). The resulting product was obtained as a slightly yellow viscous oil (40\% yield). ${ }^{1} \mathrm{H}$ NMR of CL1 (400 MHz, $\mathrm{CDCl}_{3}$, TMS standard, r.t.): $\delta=6.10$ (m; $2 \mathrm{H}$ ), $5.55(\mathrm{~m} ; 2 \mathrm{H}), 4.26(\mathrm{t} ; 4 \mathrm{H}), 3.69(\mathrm{t} ; 4 \mathrm{H}), 1.93(\mathrm{~s} ; 6 \mathrm{H}), 1.37(\mathrm{~s} ; 6 \mathrm{H})$.

CL3: We utilized a similar procedure to synthesize CL3 as described above for CL1 except substituting 5 g PEG-MA (13.89 mmol, 2.3 equiv.) in place of HEMA. The reaction was quenched by adding with $\sim 0.5 \mathrm{~mL}$ TEA $(\mathrm{pH}>7)$ and the molecular sieves were removed by filtration and solvent was evaporated in vacuum. Product was obtained as a viscous yellow liquid. By comparing the integration ratio in ${ }^{1} \mathrm{H} \mathrm{NMR}\left(\mathrm{CDCl}_{3}\right)$ spectra (see supplemental data in $\mathrm{S} 3$ ) it was confirmed that it is mixture of unreacted PEG-MA and CL3 (3.38:1). CL3, with the unfunctionalized PEG-MA impurity was used without further purification

CL2p and CL2m: $p$ MBA or $m$ MBA 5 g (36.7 mmol, 1 equiv.) and 19.1 g HEMA (146.8 mmol, 4 equiv.) were charged in a $100 \mathrm{~mL}$ round bottom flask and placed in an ice-bath in the presence of $0.49 \mathrm{~g} p$ TSA ( $2.57 \mathrm{mmol}, 0.07$ equiv.) and stirred over $4 \AA$ molecular sieves for 6 hours. The reaction was quenched by adding $\sim 1 \mathrm{~mL}$ TEA and the solution was diluted with tetrahydrofuran (THF) and molecular sieves were filtered. The crude product was purified by 
column chromatography with silica gel using a solvent mixture (hexane: dichloromethane: TEA $=85: 5: 10, \mathrm{v} / \mathrm{v})$ as the eluent. The compounds CL2p and CL2m were both obtained as colorless transparent viscous liquid in approximately $35 \%$ yield. ${ }^{1} \mathrm{H}$ NMR of CL2p (400 MHz, $\mathrm{CDCl}_{3}$, TMS standard, r.t.): $\delta=7.4(\mathrm{~d} ; 2 \mathrm{H}), 6.9(\mathrm{~d} ; 2 \mathrm{H}), 6.12(\mathrm{~m} ; 2 \mathrm{H}), 5.65(\mathrm{~s} ; 1 \mathrm{H}), 5.57(\mathrm{~m} ; 2 \mathrm{H}), 4.33(\mathrm{t}$; 4H), $3.81(\mathrm{~s} ; 3 \mathrm{H}), 3.75(\mathrm{t} ; 4 \mathrm{H}), 1.95(\mathrm{~s} ; 6 \mathrm{H}),{ }^{13} \mathrm{C}$ NMR of CL2p (100 MHz, CDCl $): \delta=167.25$, $159.77,136.15,129.87,127.94,125.71,113.58,100.97,63.70,62.68,55.24,18.30,{ }^{1} \mathrm{H}$ NMR of CL2m $\left(\mathrm{CDCl}_{3}\right): \delta=7.29(\mathrm{~d} ; 1 \mathrm{H}), 7.04(\mathrm{t} ; 2 \mathrm{H}), 6.87(\mathrm{~d} ; 1 \mathrm{H}), 6.12(\mathrm{~m} ; 2 \mathrm{H}), 5.65(\mathrm{~s} ; 1 \mathrm{H}), 5.57(\mathrm{~m}$ 2H), $4.35(\mathrm{t} ; 4 \mathrm{H}), 3.80(\mathrm{~s} ; 3 \mathrm{H}), 3.75(\mathrm{t} ; 4 \mathrm{H}), 1.95(\mathrm{~s} ; 6 \mathrm{H}),{ }^{13} \mathrm{C}$ NMR of CL2m $\left(100 \mathrm{MHz}, \mathrm{CDCl}_{3}\right)$ : $\delta=167.20,159.67,139.23,136.14,129.31,125.69,118.98,114.18,112.14,100.89,63.64,62.85$ $55.17,18.27$.

V-CL2m: A $40 \mathrm{~mL}$ round bottom flask fitted with a Dean-Stark trap filled with $25 \mathrm{~mL}$ of cyclohexane was charged with $3 \mathrm{~g}$ 5-hexen-1-ol (0.03 mol, 3 equiv.), $1.36 \mathrm{~g} \mathrm{mMBA}(0.01 \mathrm{~mol}, 1$ equiv.) and $0.02 \mathrm{~g} p$ TSA ( $0.1 \mathrm{mmol}, 0.01$ equiv.), and $40 \mathrm{~mL}$ cyclohexane. The solution was refluxed at $100{ }^{\circ} \mathrm{C}$ for $18 \mathrm{hr}$ and distilled water was periodically removed from the trap. After cooling to room temperature, the reaction was quenched by adding $\sim 1 \mathrm{~mL}$ TEA. The crude product was purified by column chromatography with silica gel using mixture of hexane: dichloromethane: TEA $(85: 5: 10, \mathrm{v} / \mathrm{v})$. as the eluent. V-CL2 was obtained as colorless transparent liquid (Yield: $80 \%$ \%) ${ }^{1} \mathrm{H}$ NMR of V-CL2m (400 MHz, $\mathrm{CDCl}_{3}$, TMS standard, r.t.): $\delta=7.3$ (t; $\left.1 \mathrm{H}\right)$, $7.07(\mathrm{~d} ; 2 \mathrm{H}), 6.89(\mathrm{~d} ; 1 \mathrm{H}), 5.84(\mathrm{~m} ; 2 \mathrm{H}), 5.50(\mathrm{~s} ; 1 \mathrm{H}), 5.0(\mathrm{~m} ; 2 \mathrm{H}), 3.84(\mathrm{~s} ; 3 \mathrm{H}), 3.58(\mathrm{t} ; 4 \mathrm{H})$, $2.09(\mathrm{~m} ; 4 \mathrm{H}), 1.64(\mathrm{~m} ; 4 \mathrm{H}), 1.51(\mathrm{~m} ; 4 \mathrm{H})$

\subsection{Measurement of acid hydrolysis of CL3}

Hydrolysis measurement were performed by adding $0.5 \mathrm{~mL}$ of buffer solutions to NMR tubes containing $0.05 \mathrm{~mL}$ of CL3. NMR spectra were taken at periodic intervals at room temperature. 
A stock solution of deuterium phosphate buffer was prepared by mixing adjusters (see supplemental S1) with a known volume of the primary salt solution and diluted with $\mathrm{D}_{2} \mathrm{O}$ to a total volume of $2 \mathrm{~mL}$. Samples were kept at room temperature in between measurements. The ratio of hydrolyzed polymer at time $t$ was determined by comparing the disappearance of the integration ${ }^{1} \mathrm{H}$ NMR spectra of the methyl protons, $\delta=1.26$ (s: $6 \mathrm{H}$ ) or appearance of acetone protons, $\delta=2.17$ (s: $6 \mathrm{H}$ ) to the methyl protons on the methacrylate group, $\delta=1.87$ (s: 18.53 ). The half-life of hydrolysis was determined as $(\ln 2) / \mathrm{k}_{\mathrm{d}}$, where $\mathrm{k}_{\mathrm{d}}$ was a negative value of the slope obtained by plotting $\ln \left([\mathrm{M}]_{\mathrm{t}} /[\mathrm{M}]_{0}\right)$ vs incubation time $t$ where $[\mathrm{M}]_{\mathrm{t}}$ and $[\mathrm{M}]_{0}$ are the relative peak integrations at time $t$ and initial time.

\subsection{Measurement of acid hydrolysis of CL2m and CL2p}

The pH-dependent hydrolysis of CL2m and CL2p was studied by observing the increase of absorbance in the UV-Vis spectra corresponding to the generation and extraction of the aromatic aldehydes, $m \mathrm{MBA}$ or $p \mathrm{MBA}$ which are the product of hydrolysis. $10 \mu \mathrm{L}$ of crosslinker solution (CL2m or CL2p in anhydrous THF at $5 \mu \mathrm{L} / \mathrm{mL}$ ) was mixed with $3 \mathrm{~mL}$ of buffer solutions of predetermined $\mathrm{pH}$ immediately before starting the absorption measurement. The intensity of the aromatic aldehyde UV absorption peak between $280-320 \mathrm{~nm}$. We arbitrarily assigned the time at $100 \%$ hydrolysis as the point where there was no further significant increase in absorbance. Absorbance was measured at different time intervals for each of the $\mathrm{pH}$ buffered solutions and used to construct the kinetic plot and calculate the half-life $\left(t_{1 / 2}\right)$ of hydrolysis. The kinetic plot was obtained by using following equation, where $A_{t}, A_{0}, A_{\infty}$ are absorbance at time $t$, initial, and theoretical 100\% hydrolysis, respectively. 


$$
-\ln \left[\frac{[\text { Crosslinker }]_{t}}{[\text { Crosslinker }]_{0}}\right]==-\ln \left[\frac{\left(A_{\infty}-A_{t}\right)}{\left(A_{\infty}-A_{0}\right)}\right]
$$

\subsection{Measurement of acid hydrolysis of crosslinked films}

Crosslinked films were prepared in $20 \mathrm{~mL}$ scintillation vials by thermally initiated polymerization of monomers and acid sensitive crosslinkers. In a typical polymerization, a vial was loaded with monomer, crosslinker (CL2m) and AIBN which had been dissolved in a small amount of diethyl ether. Polymerization was accomplished by heating the vial to $60 \sim 90{ }^{\circ} \mathrm{C}$ for 20 min under $\mathrm{N}_{2}$. After polymerization the crosslinked polymer formed a film in the bottom of the vial. The films were rinsed with hexane several times to extract unreacted monomers and any mMBA that may have formed during the reaction. The films were dried under vacuum at room temperature to remove residual ether. In the case of thiol-ene crosslinked films between V-CL2m and pentaerythritol tetrakis(3-mercaptopropionate) (2:1 molar ratio), the polymerization was conducted under $\mathrm{N}_{2}$ using UV irradiation $(365 \mathrm{~nm})$ without initiator for $15 \mathrm{~min}$. The exact film compositions are shown in the Table 1 . To study network hydrolysis each vial was filled with $\mathrm{pH}$ buffer solution $(2 \mathrm{~mL}$ ) and $2 \mathrm{~mL}$ of hexane to which $1 \mathrm{mg} / \mathrm{mL}$ of hexadecane (as an internal standard) had been added. At prescribed times $2 \mu \mathrm{L}$ of the hexane phase were taken by syringe and analyzed by GC-MS.

\section{Results and Discussion}

\subsection{Acid-sensitive crosslinkers}

The ketal crosslinkers were synthesized by reacting 2,2-dimethoxypropane (DMP) with either HEMA (yielding CL1) or PEG-MA (average $\mathrm{M}_{\mathrm{n}}: 360 \mathrm{~g} / \mathrm{mol}$ ) (yielding CL3) in the presence of a catalytic amount of $p$ TSA with or without solvent. The acetal crosslinkers were made by condensing HEMA with either $p$ MBA (yielding CL2p), or $m$ MBA (yielding CL2m) with acid 
catalysis. In the case of V-CL2m, 5-hexen-1-ol was condensed with $m \mathrm{MBA}$. In all cases care was taken to remove either the methanol or water formed during the reaction thereby favoring the formation of product and reducing the back reaction (see Figure 1). Water removal was accomplished by performing the reaction over dried molecular sieves or through in situ distillation of water or methanol and removal using a Dean-Stark trap to increase yield. ${ }^{14}$ In the case of distillation, we note that raising the reaction temperature to the boiling point of methanol or water sometimes led to an undesirable polymerization of the monomers, especially in the case of the methacrylates, giving a low yield of product. During purification by column chromatography, the slightly acidic silica gel led to undesirable hydrolysis but this could be avoided by adding small amount of TEA to the eluent. For similar reason, after column purification, it is very important to avoid evaporating the solvent at high temperature as this also leads to decomposition of the product. The monomers were kept at $0^{\circ} \mathrm{C}$ to prevent degradation or polymerization before use.

CL3 is so sensitive to hydrolysis and hydrophilic due to the PEG content that no purification was attempted after quenching the reaction with TEA and the only post-reaction processes were removal of salts and molecular sieves by filtration. ${ }^{1} \mathrm{H}$ NMR analysis reveals that the ratio of PEG-MA and CL3 was 1: 3.38. This ratio was determined by comparing integration ratio of methacrylate $\mathrm{CH}_{3}(\delta=1.9)$ and acetal $\mathrm{CH}_{3}(\delta=1.31)(\mathrm{S} 3)$. The methacrylate-based crosslinkers (CL1, CL2m, CL2p, and CL3) were all isolated in fairly low yields (20 40\%) which is inevitable due to insufficient removal of water or methanol during the reaction. However, the starting materials are not very expensive. Work continues to find ways of increasing the yields which would be especially useful when using more expensive or less available starting materials. It was also challenging to isolate the acetal products from residual mMBA or pMBA by column 
chromatography due to the very close $\mathrm{R}_{\mathrm{f}}$ values of each material. The exception was V-CL2m which was synthesized in the same manner as the other monomers except we were able to employ a Dean-Stark and run these reaction at a higher temperature to remove water instead of using molecular sieves. This effective water elimination gives higher yield (80\%) compared to low temperature reaction in the presence of molecular sieves. The resistance of 5-hexen-1-ol and V-CL2m to undergo undesrired thermal polymerization at elevated temperatures was important. V-CL2m was used for crosslinked films with multi-functional thiol monomers using the thiolene reaction and their degradation kinetics will be compared to the other methacrylate-based crosslinked films below.

\subsection{Kinetics of hydrolysis of CL3 by ${ }^{1} \mathrm{H}$ NMR}

We wish to gain an understanding of the kinetics of acid hydrolysis of the acetal-based crosslinkers in the water-based buffer solutions at certain $\mathrm{pH}$ ranges. Our interest stem from reports of the use of similar network materials under physiological conditions, a similar temperature range needed for garment applications. The hydrolysis of the HEMA-based CL1 monomer was difficult to study in aqueous buffer solutions due to its water-insolubility. To improve water solubility of the crosslinker, PEG-MA $(360 \mathrm{~g} / \mathrm{mol})$ was used to prepare the acetal CL3, using 2,2-DMP instead of HEMA during the condensation. Both CL1 and CL3 share the same acetal linking group structure.

The ${ }^{1} \mathrm{H}$ NMR degradation study of the water soluble crosslinker CL3 was conducted using a phosphate buffer solution made using deuterium oxide $\left(\mathrm{D}_{2} \mathrm{O}\right)$ with a range of $\mathrm{pH}$ from 1 to 5 and confirmed using a pH meter. ${ }^{17}$ Although a ${ }^{1} \mathrm{H}$ NMR kinetic study of CL1 wasn't conducted because of its low solubility, it can be expected that CL1 and CL3 would have different 
degradation profiles because the polarity of overall structure will affect the hydrolysis rate, and given that more hydrophilic backbones will have a higher hydrolysis rate compared to a more hydrophobic one due to solvation effects ${ }^{14}$. As an initial state $(\mathrm{t}=0),{ }^{1} \mathrm{H}$ NMR was conducted by using pure $\mathrm{D}_{2} \mathrm{O}$ at room temperature. $[\mathrm{M}]_{\mathrm{t}}$ and $[\mathrm{M}]_{0}$ are the molar concentration of ketal function at time $t$ and initial time, respectively, which were obtained using the integration of value of $\mathrm{CH}_{3}$, $\delta=1.31(\mathrm{~s} ; 6 \mathrm{H})$ (Figure 2a). The size of ${ }^{1} \mathrm{H}$ NMR peaks were monitored over time and hydrolysis rates generally increased with decreasing $\mathrm{pH}$. As shown in Figure 2b, CL3 completely decomposed in 80 minutes $\left(\mathrm{t}_{1 / 2}<14 \mathrm{~min}\right)$ at $\mathrm{pH} 5$ and $10 \min \left(\mathrm{t}_{1 / 2}<2 \mathrm{~min}\right)$ at $\mathrm{pH} 4$. At $\mathrm{pH} 1 \sim 3$, the hydrolysis was too rapid to measure by NMR as there were no observable acetal peaks to be found in the spectra. Given the time required to prepare the samples and start the NMR experiment, it is assumed that hydrolysis in these strongly acid solutions takes place in under $1 \sim$ 2 min. Lowering the $\mathrm{pH}$ results in an acceleration of the hydrolysis kinetics as the rate of acetal hydrolysis is proportional to the hydronium ion concentration. ${ }^{13}$ For some applications this rate at low $\mathrm{pH}$ may be too rapid, therefore to reduce the hydrolysis rate, acetals CL2m and CL2p based on benzaldehyde were synthesized and their degradation kinetics were also investigated.

\subsection{Degradation study of CL2m and CL2p by UV-Vis spectroscopy}

Unlike CL3, the benzaldehyde based acetals CL2m and CL2p are non-polar and insoluble in the water based buffer solution, however, both crosslinkers generate $m$ - or $p$ methoxybenzaldehyde as a result of acetal hydrolysis, which show strong UV absorbance around $300 \mathrm{~nm}$. Even using a low concentration of crosslinker $(0.016 \mu \mathrm{L} / \mathrm{mL})$, it was possible to detect small amounts of mMBA or pMBA released into buffer solutions. Samples were prepared in quartz UV cuvettes and studied in real-time by observing changes in absorption between 280 to $320 \mathrm{~nm}$ (the maximum peak values varied slightly based on the buffer $\mathrm{pH}$ ). All the UV-Vis 
spectra taken are provided as supporting information, S9 and S10. For each experiment absorbance was measured at different time intervals. It was expected that using a substituted aromatic ring with an electron donating methoxy group in the acetal position would reduce the hydrolysis rate compared to CL3. Depending on the methoxy substituent position, meta or para, hydrolysis rates were also different. The kinetic plots corresponding to CL2m (a) and CL2p (b) are shown in Figure 3 and both show accelerated hydrolysis at lower pH. Using initial slope at each $\mathrm{pH}$, the half-life of hydrolysis for samples was calculated (Figure 4) and both CL2 derivatives show a much slower rate of hydrolysis compared to $\mathrm{CL} 3$ across all $\mathrm{pH}$ ranges. At $\mathrm{pH}$ 1, there is no discernable difference measured between CL2m and CL2p ( 4 min), while at pH $2 \sim 4$, CL2m has a slightly longer half-life. This tendency is well matched with the observed trend that acetals (structures based on aldehydes) undergo slower hydrolysis than ketals (based on ketones) due to the absence of the second electron-donating methyl group to stabilize the carbocation intermediate during the hydrolysis. ${ }^{18}$

\subsection{GC-MS degradation study of network films crosslinked by acetals}

For the desired application of a reactive, self-exfoliating film, reasonable degradation rates are desired and it is important to investigate the hydrolysis rate of the network films and solve for variables such as $\mathrm{pH}$, co-monomer compositions, and crosslinking densities. In order to perform continuous measurement of network degradation we chose to look for small molecule fragments released upon hydrolysis of the acetal functionality. Crosslinked films were prepared by thermally initiated radical polymerization of methacrylate-based monomers and crosslinker CL2 $\mathrm{m}$. In the case of thiol-ene crosslinked films synthesized by the reaction of V-CL2m and pentaerythritol tetrakis(3-mercaptopropionate), polymerization was conducted under $\mathrm{N}_{2}$ without initiator under UV irradiation for $15 \mathrm{~min}$. Based on the determined degradation kinetic of the five 
crosslinkers synthesized, CL2m looked the most promising due to its reasonable hydrolysis rate above $\mathrm{pH}$ 4. When using the same crosslinker to make bulk network films, degradation rates were dictated by a combination of $\mathrm{pH}$, hydrophilicity of co-monomers, and the total crosslinking density. Due to the need to detect small amounts of degradation product when monitoring network degradation, we chose GC-MS analysis. To accomplish this we carried out hydrolysis in aqueous media and extracted the resulting $m \mathrm{MBA}$ into hexane. A known amount of hexadecane was assed to the hexane solution as an internal standard. The overall experiment is outlined in Figure 5. These experiments make some assumptions that lead to a degree of error in the measurement, such as the assumption that all mMBA generated from the hydrolysis of the acetal group would be quantitatively extracted into the hexane layer. We also make the assumption that the CL2m monomer was completely incorporated during the network polymerization and is quantitatively present in the crosslinked films. The calculated mass fraction of mMBA is $36 \mathrm{wt} \%$ in the CL2m network or $42 \mathrm{wt} \%$ in the V-CL2m networks. A calibration curve was made using standard solutions of mMBA by plotting response ratio versus amount ratio (see supplemental S11). The response ratio is defined as ratio of area of the mMBA peak and area of the hexadecane (an internal standard) peak. Amount ratio is defined as ratio of amount of mMBA and amount of hexadecane.

In the case of crosslinked films composed of HEMA and CL2m (30:1 molar ratio - $3.2 \mathrm{~mol} \%$ of CL2m), $\ln \left([\mathrm{M}]_{\mathrm{t}} /[\mathrm{M}]_{0}\right)$ in different $\mathrm{pH}$ buffers was plotted as a function of exposure time and the half-lives were determined (Figure 6). The rates of hydrolysis were slow, mMBA was not detected for the first $21 \mathrm{hr}$ when reacted at $\mathrm{pH} 1$ and $\mathrm{pH} 2$. A small amount of hydrolysis may have started before this time but detectable amounts of mMBA extracted into hexane were detected by GC-MS only after $21 \mathrm{hr}$. Still, the plots show an increase of mMBA over time (see supplemental 
S12). As the acidity of the solutions was reduced, the hydrolysis dropped precipitously and even after 8 days, we were unable to detect any mMBA at pH5. This indicates that these crosslinked film have much slower degradation rate at $\mathrm{pH} 5$ compared to $\mathrm{pH} 1 \sim 4$. The first order reaction plots were constructed (Figure 6a) and corresponding half-life time at each $\mathrm{pH}$ was obtained using initial slope. The calculated half-lives were $25 \mathrm{hr}, 33 \mathrm{hr}, 160 \mathrm{hr}$ and $533 \mathrm{hr}$ at pH1, 2, 3 and 4 respectively. These results make it clear that the hydrolysis rates of the acetal groups are much slower after incorporation into networks.

Interestingly, if the same CL2m crosslinker concentration is used, but the commoner is MMA rather than HEMA, the resulting networks fail to show any measurable hydrolysis (see supplemental S13). This is because MMA imparts a much more hydrophobic nature to the network slowing diffusion of aqueous buffer and hence lowering the observed rate of hydrolysis. Control of rate of hydrolysis should be selectable by controlling the relative amounts of hydrophilic or hydrophobic comonomers in the network.

Another variable that can be adjusted to control hydrolysis rate is crosslinking density (Figure 7). The crosslinking density can be increased or decreased by varying the concentration of CL2m used in the polymerization. The rate of hydrolysis as a function of crosslinking density was examined in pH1 buffer solution. The half-life of hydrolysis for each film was $25 \mathrm{hr}, 125 \mathrm{hr}, 521$ $\mathrm{hr}$ and $713 \mathrm{hr}$ at $3.2 \mathrm{~mol} \%, 9 \mathrm{~mol} \%, 50 \mathrm{~mol} \%$ and $66 \mathrm{~mol} \%$, respectively. As expected, the more densely crosslinked films show much slower hydrolysis rate. The buffer solution penetrates, swells, and diffuses much more slowly in the more densely crosslinked films. The half-life of acetal hydrolysis was found to be linearly proportional to the mol\% of CL $2 \mathrm{~m}$ in the films.

All of the networks described to this point were made through free radical chain polymerization processes. We wished to explore step-growth polymerized crosslinked films 
where the thiol-ene bond formation proceeds via the radical addition reaction between the divinyl monomer (V-CL2m) and a tetra-functional thiol crosslinker (PTMPA). To maximize molecular weight, thiol-ene step-growth polymerization mechanism requires exact stoichiometry between available ene groups and thiol groups. In the case of V-CL2m, $66 \mathrm{~mol} \%$ is required (33 mol\% PTMPA). The crosslinked reaction was facilitated using UV curing (365 nm) without initiator under $\mathrm{N}_{2}$ at room temperature. After initiation, the reaction proceeds quickly yielding solid, network films. Subsequent acid catalyzed hydrolysis of this resin results in depolymerization and the formation of mMBA (transparent liquid) and a four-armed, star alcohol, pentaerythritol tetrakis(3-(6-hydroxyhexylthio)propanoate) (888.42 g/mol) (Figure 8). Unlike methacrylate-based crosslinked films which retain their shapes during the hydrolysis, we could observe a transition to a viscous flowing material during the hydrolysis of the thiol-ene network. The presence of mMBA in the hexane phase was confirmed by GC-MS, which was used to examine hydrolysis kinetics.

The degradation kinetics of the thiol-ene crosslinked films were examined at different $\mathrm{pH}$ ranges. Compared to HEMA-based networks, the thiol-ene crosslinked films are more hydrophobic due to absence of free hydroxyl groups, or PEG-like segments in the network and a relatively larger portion of alkyl chains. The thiol-enes are also densely crosslinked, hindering swelling and diffusion of buffer solution. At $\mathrm{pH} 1$ and 2, physical decomposition of the films was visually observed and parts of the region began to flow after $96 \mathrm{hr}$ in buffer. The half-life at each

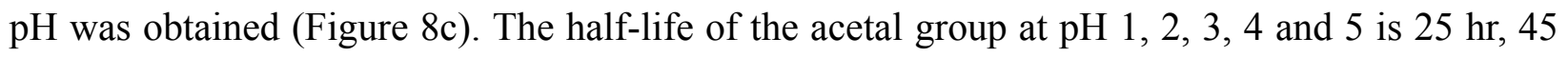
$\mathrm{hr}, 106 \mathrm{hr}, 460 \mathrm{hr}$ and $837 \mathrm{hr}$, respectively. The thiol-ene networks had a much shorter $\mathrm{t}_{1 / 2}$ as compared to the HEMA/CL2m films at $\mathrm{pH} 1\left(\mathrm{t}_{1 / 2}=713 \mathrm{hr}\right)$ even they have same mol $\%$ of crosslinker. Essentially all decomposition during hydrolysis of the thiol-ene polymers converts 
high molecular weight polymer chains directly to low molecular weight residues, a tetrafunctioned molecule and mMBA (Figure 8a). This results in increased diffusion and permeation of buffer solution, which in turn, continues to increase the rate of depolymerization. Conversely, the HEMA/CL2m films still have partially crosslinked or entangled linear polymer during the hydrolysis because the polymer interconnected structure that was formed via chain-growth polymerization contains a broad range of number of repeat units between crosslink junctions. We propose that this difference results in the faster hydrolysis observed in the hydrophobic thiol-ene crosslinked films with at the same crosslinker mol\%. The vinyl terminated monomer V-CL2m has merits since it can be synthesized in high yield, however, it cannot be utilized in conventional free radical polymerizations do to the low reactivity of the vinyl group. Therefore its use in network formation is limited to reactions such thiol-ene step polymerization. Hence, selection of appropriate thiol comonomers becomes important and in this study only one was used. This leaves room for improvement in this area and is currently under investigation.

\section{Conclusions}

Through various acid-sensitive acetal and ketal-based groups with different substituents and adjusting the degree of hydrophilicity of the crosslinker unit, we demonstrate chemically tuned molecules that show different room temperature hydrolysis rates at different $\mathrm{pH}$ ranges. In the case of 2,2'-DMP-based acetal groups, CL3 revealed the highest acid-sensitivity $\left(\mathrm{t}_{1 / 2}<14 \mathrm{~min}\right.$ at $\mathrm{pH} 5$ and $\mathrm{t}_{1 / 2}<2 \min$ at $\left.\mathrm{pH} 4\right)$. Acetal functional groups based on meta or para methoxybenzaldehydes (CL2m or CL2p) gave crosslinkers with a reduced hydrolysis. By polymerizing CL2m various comonomers (HEMA or MMA), networks were synthesized and their hydrolysis rates were measured as a function of $\mathrm{pH}$, network hydrophobicity, and crosslinking density. When the vinyl-terminated crosslinker (V-CL2m) was reacted with a 
tetrathiol, thiol-ene networks were prepared. This densely crosslinked, hydrophobic polymer exhibited a much faster hydrolysis rate than HEMA/CL3 based networks with a comparable crosslinking density. These materials are being studied as coatings for protective garments resistant to chemical warfare agents. This basic study of hydrolysis and degradation kinetics of acid-sensitive monomers and networks provides parameters needed to use these materials as stimuli responsive materials. A companion study currently underway in collaboration with the US Army Natick Soldier RD\&E Center is examining the kinetics of neutralization of chemical warfare agents within these networks and the moisture vapor transport properties of thin network films. These materials may have additional utility in controlled release applications, advanced patternable resists and a number of other areas where such control of network stability is desired.

\section{Acknowledgements}

The authors acknowledge the financial support of the Chemical and Biological Technologies Department of the Defense Threat Reduction Agency (DTRA-CB) via grant BA12PHM123 in the "Dynamic Multifunctional Materials for a Second Skin D[MS]2" program. 


\section{Figure Legends}

Table 1. Acid-degradable film compositions (molar ratio), hydrolysis solution $\mathrm{pH}$, and $\mathrm{t}_{1 / 2}$ of crosslinker.

Figure 1. Synthesis of acid-sensitive crosslinker monomers

Figure 2. Acid catalyzed degradation of CL3: (a) ${ }^{1} \mathrm{H}$ NMR spectra before and after degradation, and (b) the corresponding hydrolysis kinetics at $\mathrm{pH} 4$ and 5 versus time.

Figure 3. Acid catalyzed degradation of (a) CL2m and (b) CL2p at pH1, 2, 3 and 4.

Figure 4. Plots of the half-life of CL2m and CL2p during hydrolysis at various $\mathrm{pH}$.

Figure 5. (a) Hydrolysis of CL2m-based networks and (b) schematic representation of network degradation experiment for the GC-MS study.

Figure 6. (a) Plot of $\ln \left([\mathrm{M}]_{\mathrm{t}} /[\mathrm{M}]_{0}\right)$ in different buffer solutions versus incubation time and (b) plot of the half-life of crosslinkers in the network as a function of $\mathrm{pH}$.

Figure 7. (a) Plot of $\ln \left([\mathrm{M}]_{\mathrm{t}} /[\mathrm{M}]_{0}\right)$ for networks with different crosslinker concentration versus incubation time and (b) plot of the half-life of crosslinkers in the network as a function of crosslinker concentration.

Figure 8. Thiol-ene networks: (a) hydrolysis of thiol-ene based networks, (b) plot of $\ln \left([\mathrm{M}]_{\mathrm{t}} /[\mathrm{M}]_{0}\right)$ in different buffer solutions versus incubation time and (c) plot of the half-life of crosslinkers in the network as a function of $\mathrm{pH}$. 
Table 1. Acid-degradable film compositions (molar ratio), hydrolysis solution $\mathrm{pH}$, and $\mathrm{t}_{1 / 2}$ of crosslinker.

\begin{tabular}{|c|c|c|c|}
\hline Monomer & Crosslinker & pH & $\mathbf{t}_{1 / 2}$ \\
\hline HEMA & CL2m & & \\
\hline \multirow{5}{*}{30} & \multirow{5}{*}{$1(3.2 \mathrm{~mol} \%)$} & 5 & Non-measurable \\
\hline & & 4 & $533 \mathrm{hr}$ \\
\hline & & 3 & $160 \mathrm{hr}$ \\
\hline & & 2 & $33 \mathrm{hr}$ \\
\hline & & \multirow{4}{*}{1} & $25 \mathrm{hr}$ \\
\hline 10 & $1(9 \mathrm{~mol} \%)$ & & $125 \mathrm{hr}$ \\
\hline 1 & $1(50 \mathrm{~mol} \%)$ & & $521 \mathrm{hr}$ \\
\hline 0.5 & $1(66 \mathrm{~mol} \%)$ & & $713 \mathrm{hr}$ \\
\hline MMA & CL2m & & \\
\hline 30 & $1(3.2 \mathrm{~mol} \%)$ & $1 \sim 5$ & infinite \\
\hline 4-thiol & V-CL2m & & \\
\hline \multirow{5}{*}{1} & \multirow{5}{*}{$2(66 \mathrm{~mol} \%)$} & 5 & $837 \mathrm{hr}$ \\
\hline & & 4 & $460 \mathrm{hr}$ \\
\hline & & 3 & $106 \mathrm{hr}$ \\
\hline & & 2 & $45 \mathrm{hr}$ \\
\hline & & 1 & $25 \mathrm{hr}$ \\
\hline
\end{tabular}




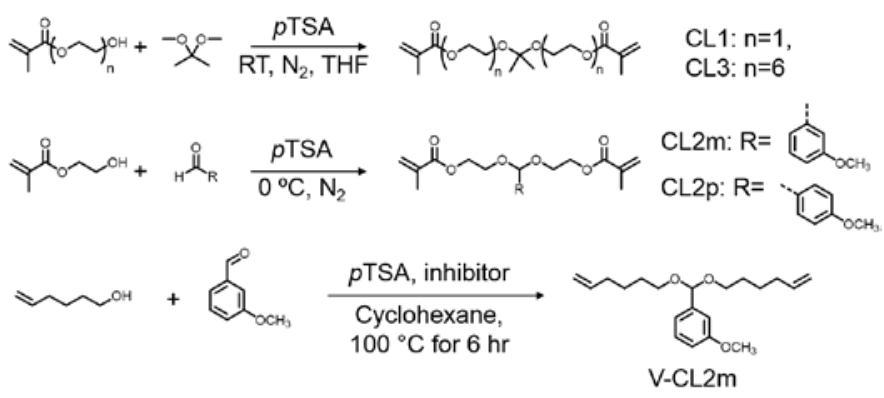

Figure 1. Synthesis of acid-sensitive crosslinker monomers 
(a)
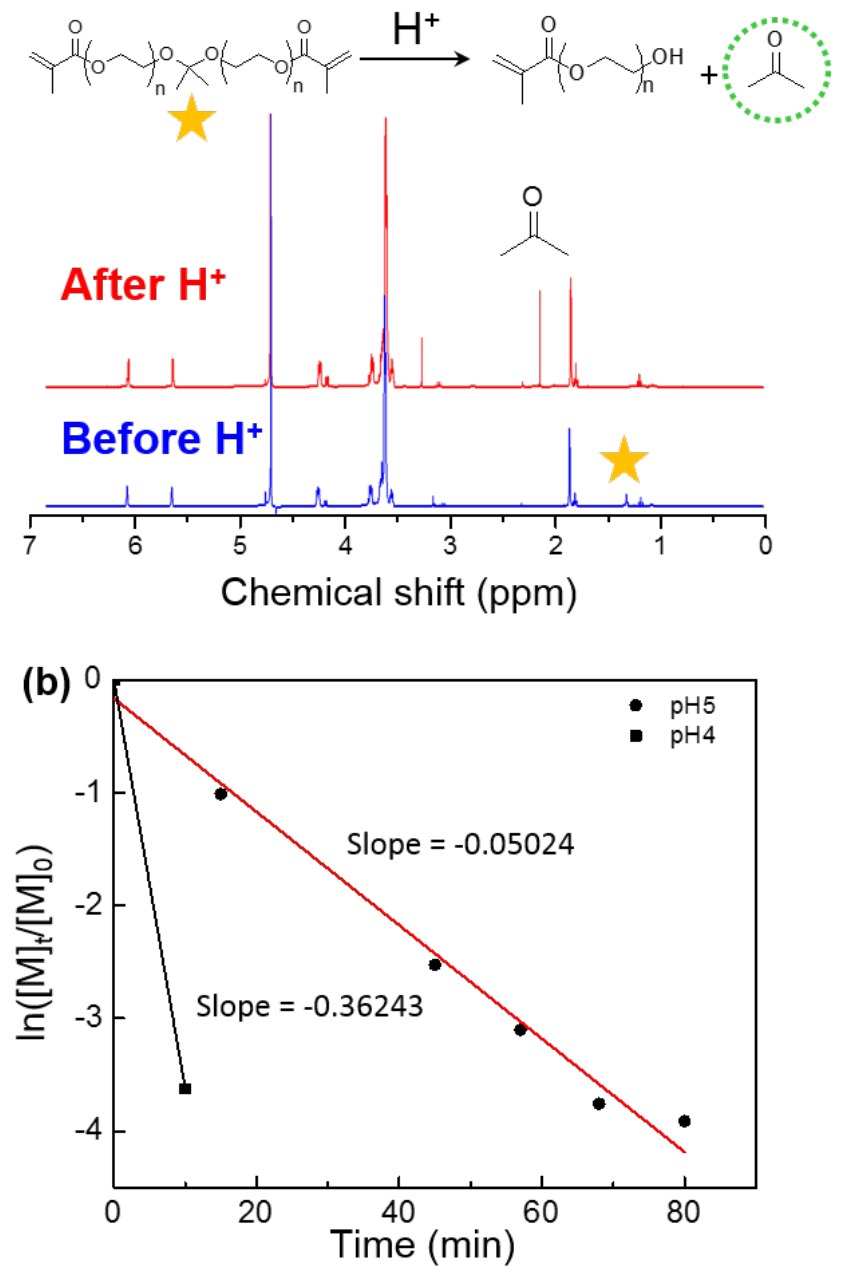

Figure 2. Acid catalyzed degradation of CL3: (a) ${ }^{1} \mathrm{H}$ NMR spectra before and after degradation, and (b) the corresponding hydrolysis kinetics at $\mathrm{pH} 4$ and 5 versus time. 

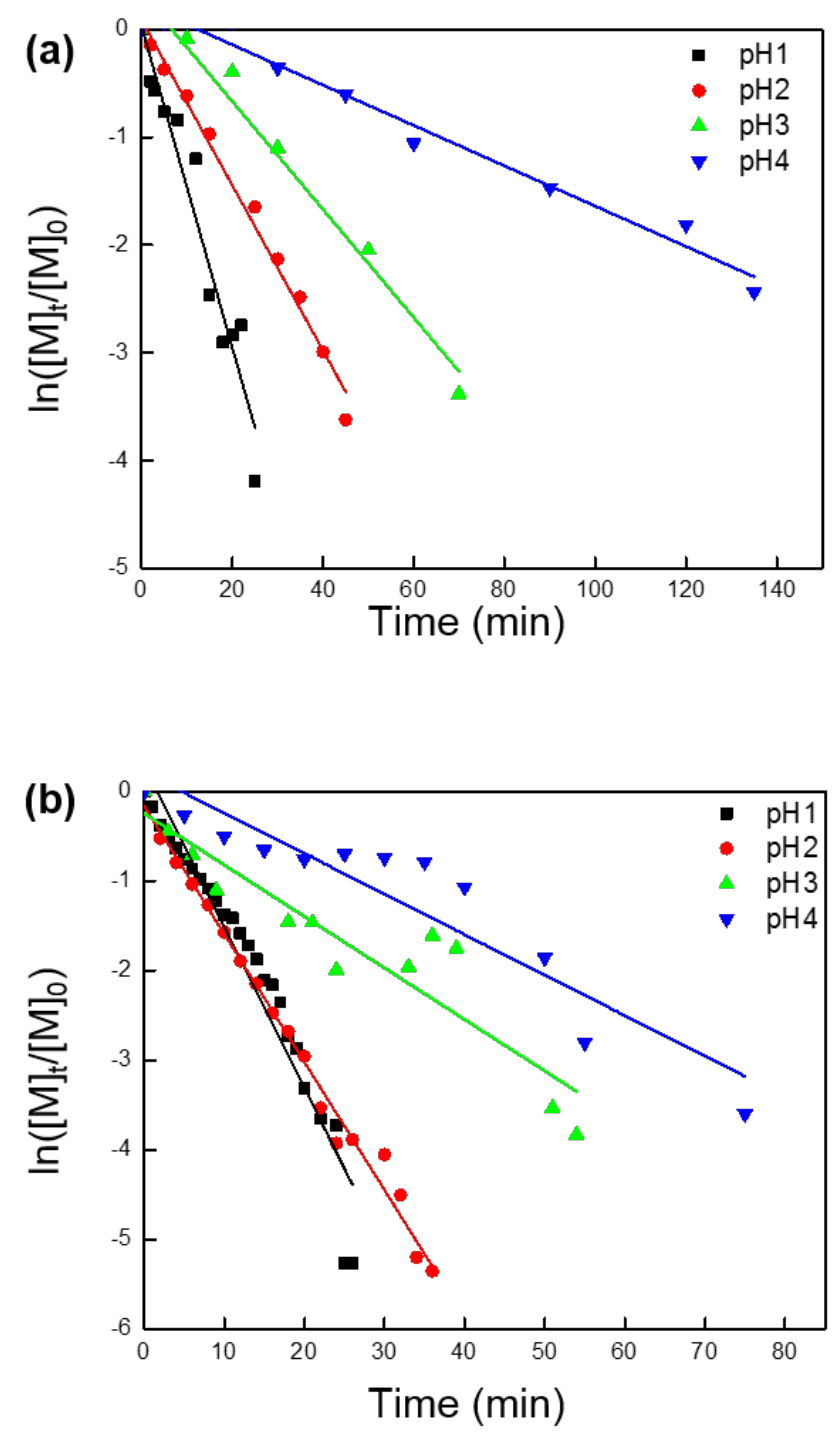

Figure 3. Acid catalyzed degradation of (a) CL2m and (b) CL2p at pH1, 2, 3 and 4. 


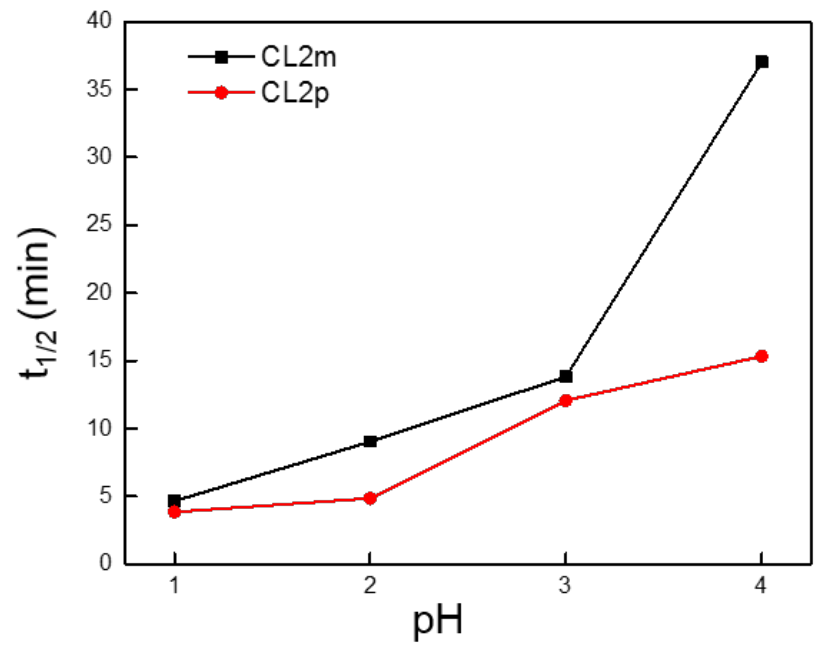

Figure 4. Plots of the half-life of CL2m and CL2p during hydrolysis at various $\mathrm{pH}$. 
(a)

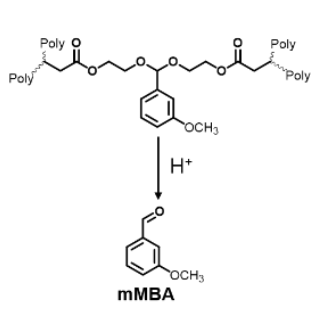

(b)

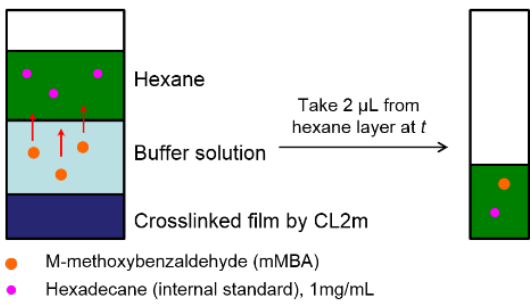

Figure 5. (a) Hydrolysis of CL2m-based networks and (b) schematic representation of network degradation experiment for the GC.MS study. 

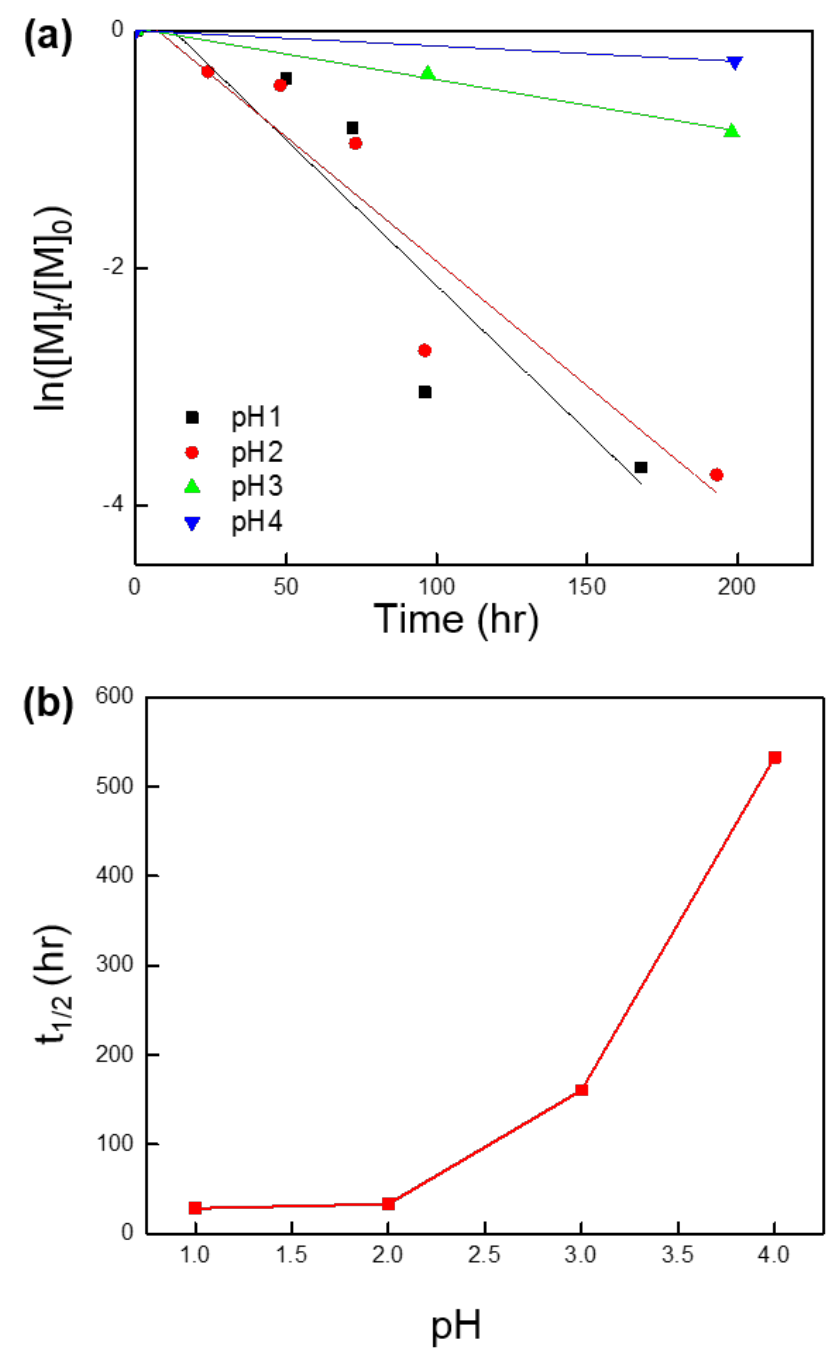

Figure 6. (a) Plot of $\ln \left([\mathrm{M}]_{\mathrm{t}} /[\mathrm{M}]_{0}\right)$ in different buffer solutions versus incubation time and (b) plot of the half-life of crosslinkers (CL2m) in the network as a function of $\mathrm{pH}$. 

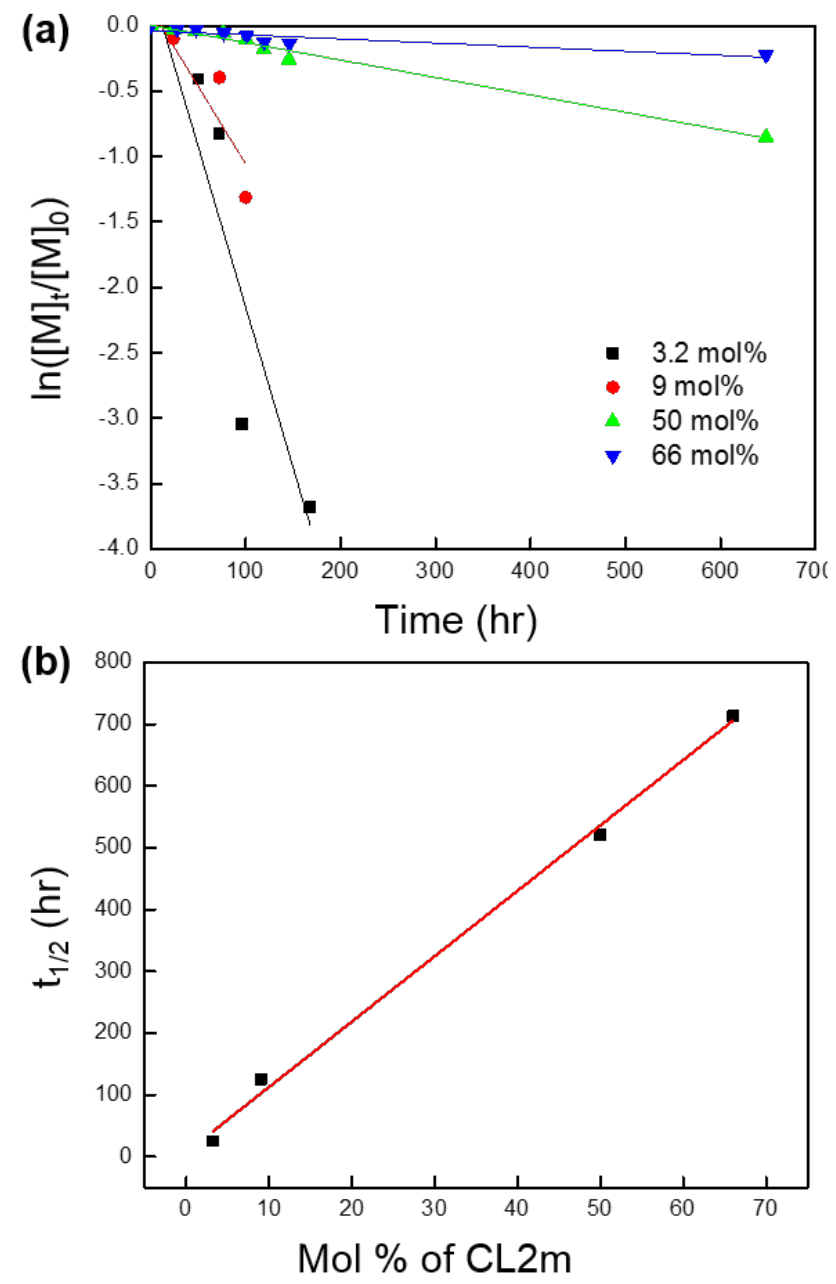

Figure 7. (a) Plot of $\ln \left([\mathrm{M}]_{\mathrm{t}} /[\mathrm{M}]_{0}\right)$ for networks with different crosslinker concentration versus incubation time and (b) plot of the half-life of crosslinkers (CL2m) in the network as a function of crosslinker concentration. 
(a)

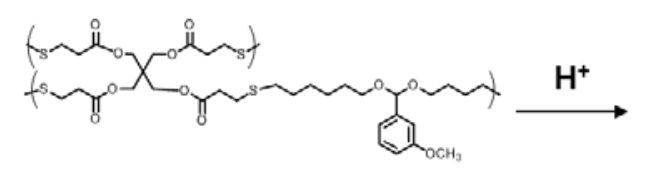

" hydrophobic, densely crosslinked"

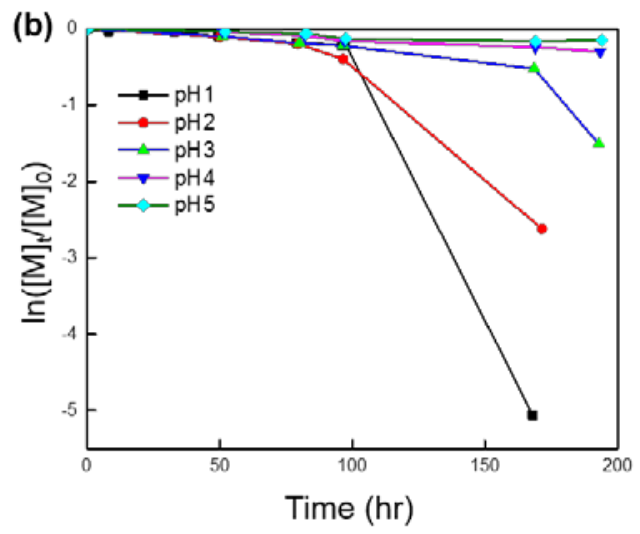

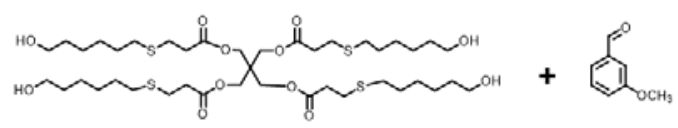

"depolymerized adducts"

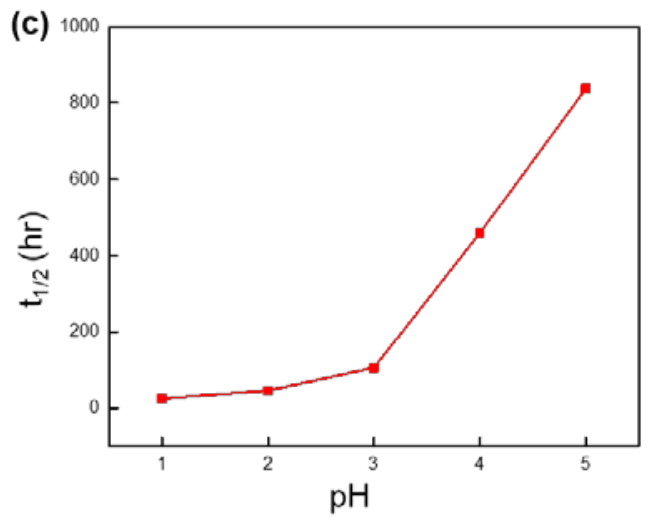

Figure 8. Thiol-ene networks: (a) hydrolysis of thiol-ene based networks, (b) plot of $\ln \left([\mathrm{M}]_{\mathrm{t}}[\mathrm{M}]_{0}\right)$ in different buffer solutions versus incubation time and (c) plot of the half-life of crosslinkers in the network as a function of $\mathrm{pH}$. 


\section{References:}

1. Murthy, N.; Thng, Y. X.; Schuck, S.; Xu, M. C.; Fréchet, J. M. J., "A Novel Strategy for Encapsulation and Release of Proteins: Hydrogels and Microgels with Acid-Labile Acetal CrossLinkers." Journal of the American Chemical Society 2002, 124 (42), 12398-12399.DOI: $10.1021 / \mathrm{ja} 026925 \mathrm{r}$

2. Khaja, S. D.; Lee, S.; Murthy, N., "Acid-Degradable Protein Delivery Vehicles Based on Metathesis Chemistry." Biomacromolecules 2007, 8 (5), 1391-1395.DOI: 10.1021/bm061234z

3. Wang, Y.; Chang, B. S.; Yang, W. L., "pH-Sensitive Polyketal Nanoparticles for Drug Delivery." J. Nanosci. Nanotechnol. 2012, 12 (11), 8266-8275.DOI: 10.1166/jnn.2012.6677

4. Paramonov, S. E.; Bachelder, E. M.; Beaudette, T. T.; Standley, S. M.; Lee, C. C.; Dashe, J.; Frechet, J. M. J., "Fully Acid-Degradable Biocompatible Polyacetal Microparticles for Drug Delivery." Bioconjugate Chem. 2008, 19 (4), 911-919.DOI: 10.1021/bc7004472

5. $\quad$ Knorr, V.; Russ, V.; Allmendinger, L.; Ogris, M.; Wagner, E., "Acetal Linked Oligoethylenimines for Use as pH-Sensitive Gene Carriers." Bioconjugate Chem. 2008, 19 (8), 1625-1634.DOI: $10.1021 / \mathrm{bc} 8001858$

6. Kim, B.; Lee, E.; Kim, Y.; Park, S.; Khang, G.; Lee, D., "Dual Acid-Responsive Micelle-Forming Anticancer Polymers as New Anticancer Therapeutics." Advanced Functional Materials 2013, 23 (40), 5091-5097.DOI: 10.1002/adfm201300871

7. Binauld, S.; Stenzel, M. H., "Acid-Degradable Polymers for Drug Delivery: A Decade of Innovation." Chem. Commun. 2013, 49 (21), 2082-2102.DOI: 10.1039/c2cc36589h

8. Palmieri, F.; Adams, J.; Long, B.; Heath, W.; Tsiartas, P.; Willson, C. G., "Design of Reversible Cross-Linkers for Step and Flash Imprint Lithography Imprint Resists." ACS Nano 2007, 1 (4), 307-312.DOI: 10.1021/nn7001079

9. Heath, W. H.; Palmieri, F.; Adams, J. R.; Long, B. K.; Chute, J.; Holcombe, T. W.; Zieren, S.; Truitt, M. J.; White, J. L.; Willson, C. G., "Degradable Cross-Linkers and Strippable Imaging Materials for Step-and-Flash Imprint Lithography." Macromolecules 2008, 41 (3), 719-726.DOI: 10.1021/ma702291k

10. Zhou, H.; Blackwell, J. M.; Lee, H. B. R.; Bent, S. F., "Highly Sensitive, Patternable Organic Films at the Nanoscale Made by Bottom-up Assembly." ACS Appl. Mater. Interfaces 2013, 5 (9), 3691-3696.DOI: 10.1021/am4002887

11. Jhaveri, S. B.; Carter, K. R., "Triggered Decomposition of Polymeric Nanoparticles." Macromolecules 2007, 40 (22), 7874-7877.DOI: 10.1021/ma071750x

12. Koylu, D.; Carter, K. R., "Stimuli-Responsive Surfaces Utilizing Cleavable Polymer Brush Layers." Macromolecules 2009, 42 (22), 8655-8660.DOI: 10.1021/ma901627c

13. Jain, R.; Standley, S. M.; Fréchet, J. M. J., "Synthesis and Degradation of pH-Sensitive Linear Poly(Amidoamine)S." Macromolecules 2007, 40 (3), 452-457.DOI: 10.1021/ma062319v

14. Wang, Y.; Morinaga, H.; Sudo, A.; Endo, T., "Synthesis of Amphiphilic Polyacetal by Polycondensation of Aldehyde and Polyethylene Glycol as an Acid-Labile Polymer for Controlled Release of Aldehyde." Journal of Polymer Science Part A: Polymer Chemistry 2011, 49 (3), 596602.DOI: $10.1002 /$ pola.24425

15. Tomlinson, R.; Klee, M.; Garrett, S.; Heller, J.; Duncan, R.; Brocchini, S., "Pendent Chain Functionalized Polyacetals That Display pH-Dependent Degradation: A Platform for the Development of Novel Polymer Therapeutics." Macromolecules 2002, 35 (2), 473-480.DOI: 
10.1021/ma0108867

16. Wang, K.; Lu, J.; Yin, R.; Chen, L.; Du, S.; Jiang, Y.; Yu, Q., "Preparation and Properties of Cyclic Acetal Based Biodegradable Gel by Thiol-Ene Photopolymerization." Materials Science and Engineering: C 2013, 33 (3), 1261-1266.DOI: http://dx.doi.org/10.1016/j.msec.2012.12.024

17. Shimoda, A.; Sawada, S.; Kano, A.; Maruyama, A.; Moquin, A.; Winnik, F. M.; Akiyoshi, K., "Dual Crosslinked Hydrogel Nanoparticles by Nanogel Bottom-up Method for SustainedRelease Delivery." Colloids and Surfaces B-Biointerfaces 2012, 99, 38-44.DOI: 10.1016/j.colsurfb.2011.09.025

18. Gillies, E. R.; Goodwin, A. P.; Frechet, J. M. J., "Acetals as pH-Sensitive Linkages for Drug Delivery." Bioconjugate Chem. 2004, 15 (6), 1254-1263.DOI: 10.1021/bc049853x 